\title{
Exchange Rate Model Approximation, Forecast and Sensitivity Analysis by Neural Networks, Case Of Iran
}

\author{
Dr. Mehdi Pedram \\ Department of Economics, Al-Zahra University \\ PO Box 1993891176, Vanak, Tehran, Tehran Province, Iran \\ Tel: 98-8805-8940Ｅ-mail: Mehdipedram@alzahra.ac.ir \\ Maryam Ebrahimi (Corresponding author) \\ Department of Economics, Al-Zahra University \\ PO Box 1993891176, Vanak, Tehran, Tehran Province, Iran \\ Tel: 98-8805-8940Ｅ-mail: Maryam.ebrahimi2000@Gmail.com
}

Received: July 1, 2014 Accepted: July 15, 2014

doi:10.5296/ber.v4i2.5892 URL: http://dx.doi.org/10.5296/ber.v4i2.5892

\begin{abstract}
This paper investigates the model estimation and data forecasting of exchange rate using artificial neural network. Recent studies have shown the classification and prediction power of the neural networks. It has been demonstrated that a neural network can approximate any continuous function. Here, in a technical approach, it has been used ARIMA and neural network for a short-term forecast of daily USD to Rial exchange rate. ANN is employed in training and learning processes and thereafter the forecast performance measured making use of two common loss functions. The comparison demonstrates that neural network is far better than ARIMA, the error is about the half.

Thereafter, in a fundamental approach via another neural network the effects of some of the most important economic variables on exchange rate prediction in a long-term sense are studied. By sensitivity analysis, the importance and the weight of each economic variable on exchange rate has determined. The results show that it is possible to estimate a model to forecast the value of exchange rate even by having access to a limited subset of data.
\end{abstract}

Keywords: Exchange rate, Forecast, Model approximation, Crude oil, Gold, Price index, Sensitivity analysis. 


\section{Introduction}

Forecasting is a very important and essential tool in the financial markets for various players such as investors, academicals, practitioners, regulators and policy makers. Forecasting with a weak set of tools and models will lead to taking wrong judgments which has an adverse effect on decision making process.

Exchange rate is one of the most valuable parameters on governmental financial and monetary policies. So an accurate forecasting model for this variable has drawn much academic and decision maker's interest. In the last four or five decades many different models have been proposed in the literature to model and forecast exchange rates.

It is an established fact that increased volatility of a variable and using weak forecasting technique in the financial markets is harmful to economic development due to their adverse impact on international trade and foreign investment (Chang and Foo, 2002). Hence, forecasting a variable in the financial markets is a matter of imperative importance, especially in a country like Iran.

Various econometric models are readily available to forecast a variable in the financial markets. In particular, Autoregressive Integrated Moving Average (ARIMA) is an extensive model of time series forecasting in which past observation of same variable are collected and analyzed to develop a model describing the relationship and then used to extrapolate the time series into the future. One of the limitations associated with this model is the disability of capturing the non-linear pattern of the time series variable (Timmermann and Granger, 2004). Hence classical statistical and econometric models used for forecasting in the field of financial time series fails to efficiently handle uncertainty nature of foreign exchange data series.

Neural networks have an advantage over traditional methods in approximation of nonlinear behaviors. An artificial neural network (ANNs), as an emerging discipline emulates the information processing capabilities of neurons of the human brain. It uses a distributed representation of the information stored in the network, and thus resulting in robustness against damage and corresponding fault tolerance (Shadbolt and Taylor, 2002).

ANNs are nowadays useful in a large variety of modeling and forecasting problems. To develop a feed forward artificial neural network for forecasting exchange rate purpose, the specification of its architecture in terms of number of inputs, number of hidden layer, number of neurons and output is very important.

In empirical studies, Kamruzzan \& Sarker (2003) use ANNs to predict six currencies against the Australian dollar and comparison it to ARIMA model. They show that the ANNs outperformed the ARIMA model in every case for each of the six currencies. Rudra P. Pradhan (2010) employs Artificial Neural Network (ANN) to forecast foreign exchange rate in India during 1992-2009. Empirical results confirm that ANN is an effective tool to forecast the exchange rate. AMM. Jamal (2011) applies the neural network model to forecast bilateral exchange rates between U.S. and Germany and U.S. and France. The predictions from the neural network model were compared to those based on a standard econometric model. The 
results suggest that the neural network model may have some advantages when frequent short term forecasts are needed. The results of Adam Stokes (2011) researches show that ANNs are able to deal with daily and weekly data as well as the nonlinearities in exchange rate movements. But Vincenzo Pacelli (2012) by empirically comparing mathematical models developed in his research, investigate that the ARCH and GARCH models, especially in their static formulations, are better than the ANN for analyzing and forecasting the dynamics of the exchange rates. Georgios Sermpinis \& et al (2012) in their research has introduced a hybrid Neural Network architecture of Particle Swarm Optimization and Adaptive Radial Basis Function (ARBF-PSO), a time varying leverage trading strategy based on Glosten, Jagannathan and Runkle (GJR) volatility forecasts and a Neural Network fitness function for financial forecasting purposes. They believe that the ARBF-PSO architecture outperforms all other models in terms of statistical accuracy and trading efficiency for the three exchange rates. Akintunde Mutairu Oyewale (2013) in his study investigates the modeling, description and forecasting of exchange rates of four countries using Artificial Neural Network. He yields that the ANN is a very effective tool for exchange rate forecasting of financial time series due to non-linearity, non-stationary and high degree of noise. Present paper uses Artificial Neural Network as an alternative model for forecasting exchange rate in Iran in both technical and fundamental approaches.

\section{Methodology}

In this section, we collect some useful methods which are used to forecast economic variables. Usually there are two approaches to forecast the future values of exchange rate. The first one is technical which is based on past behavior of the variable. In this way we assume -which is basically true- the past observations of the variable, contain mostly all of the information are needed to forecast. So we do not want to detect the causal relationships between this variable and the other ones. ARIMA is an example of this approach.

In Fundamental approach the forecast is based on an estimated model which contain the relations between the variable of interest and other economic variables. Purchasing power parity, Monetary and Portfolio balance models are some examples of this approach.

\section{ARIMA: Auto Regressive Integrated Moving Average}

Time series models use the past movements of variables in order to predict their future values. Unlike structural models that relate a main variable to a set of other variables, the time series model is not based on economic theory. However, in term of forecasting, the reliability of the estimated equation should be based on out-of-sample performance (Stock and Watson, 2003). The time series model can mostly produce quite accurate forecasts, especially in case that there are multidimensional relationships among variables. Usually because of the complexity of economic variables relations, structural models are likely to suffer from omitted variable bias, misspecifications, simultaneous causality and other problems leading to substantial forecasting errors (Keck, Raubold and Truppia, 2009).

One of widely used time series models is ARIMA. These models are chosen rather than the others such as Average Moving, Average Naïve, due to its flexibility that it can represent several different types of time series, i.e. pure autoregressive (AR), pure moving average (MA) 
and combined AR and MA (ARMA) series. The ARIMA model is denoted by ARIMA $(p, d, q)$, where " $p$ " stands for the order of the auto regressive process, " $d$ " is the order of the data stationary and " $q$ " is the order of the moving average process (Wan Khade, 2010). In ARIMA model, the future value of a variable is assumed to be a linear function of several past observations and random errors. That is, the underlying process that generate the time series has the form

$$
Y_{t}=\theta_{0}+\emptyset_{1} Y_{t-1}+\emptyset_{2} Y_{t-2}+\cdots+\emptyset_{p} Y_{t-p}+\varepsilon_{t}-\theta_{1} \varepsilon_{t-1}-\theta_{2} \varepsilon_{t-2}-\cdots-\theta_{l} \varepsilon_{t-l}
$$

Where $Y_{t}$ and $\varepsilon_{t}$ are the actual value and random error at time period $t$, respectively; $\emptyset_{i}(i=1$, $2 \ldots p)$ and $\theta_{j}(j=0,1,2 \ldots q)$ are model parameters. The integer's $\mathrm{p}$ and $\mathrm{q}$ are often referred to as orders of the model. Random errors, $\varepsilon_{t}$ are assumed to be independently and identically distributed with a mean of zero and a constant variance of $\sigma^{2}$. Equation (1) entails several important special cases of the ARIMA family of models. If $q=0$, then (1) becomes an AR model of order $\mathrm{p}$. When $p=0$, the model reduces to an MA model of order $q$. One central task of the ARIMA model building is to determine the appropriate model order $(p, q)$ (Zhang, 2003). In ARIMA stages there is more detail step from choosing model until forecasting step; it is called Box-Jenkins methodology for forecasting. The time series model using Box- Jenkins approach has been proposed by Box and Jenkins (1970). This approach has been widely used in literatures because of its performance and simplicity.

The time series used in this paper contain values of USD to Rials daily exchange rate over the time period of Nov 2010 to June 2013, totally 977 values. Eviews v8 software is used in this research.

The variable is first checked for its unit root properties using the Augmented Dickey Fuller (ADF) unit root tests. Results of this test is displayed in table 1. As the ADF has non-stationary null hypothesis, it generally suggest the variable is integrated of order one. In other words, it is non-stationary in level but is stationary of first difference or I (1).

Based on Box-Jenkins methodology it is necessary to go through 4 steps. First of all in model identification and model selection, the $(\mathrm{p}, \mathrm{d}, \mathrm{q})$ parameters must be determined. Here by checking auto correlation and partial auto correlation chart, it can be said a good guess for $(p, d, q)$ is $\operatorname{ARIMA}(1,0,0)$. It is obvious that a $\operatorname{ARIMA}(1,0,0)$ is identical to $\operatorname{ARIMA}(0,1,0)$, so according to correllogram chart the model is $\operatorname{ARIMA}(0,1,0)$.

Table 1. the result of augmented Dickey-Fuller test

\begin{tabular}{|c|c|c|c|c|c|}
\hline \multirow{2}{*}{ State } & \multicolumn{3}{|c|}{ Critical Values } & \multirow{2}{*}{ Prob. } & \multirow{2}{*}{ t statistics } \\
\cline { 2 - 4 } & $1 \%$ & $5 \%$ & $10 \%$ & & -2.044 \\
\hline intercept and trend & -3.96 & -3.41 & -3.12 & 0.57 & 0.57 \\
\hline intercept & -3.43 & -2.86 & -2.56 & 0.87 & 0.28 \\
\hline Without intercept and trend & -2.56 & -1.94 & -1.61 & 0.94 & 1.28 \\
\hline
\end{tabular}


For diagnostic purposes, it's good to examine some variations in the model which is determined in last steps by adding some more moving average and autoregressive terms. $\operatorname{ARIMA}(2,0,0), \operatorname{ARIMA}(2,0,1), \operatorname{ARIMA}(2,0,2), \operatorname{ARIMA}(1,0,2), \operatorname{ARIMA}(3,0,2)$ are tested. While ARIMA $(3,0,2)$ is better in Durbin-Watson criteria, still $\operatorname{ARIMA}(1,0,0)$ is the best among all of tested variations. After parameter estimation, the next step is using computation algorithms to arrive at coefficients which are best fitted to selected ARIMA model.

Table 2. the result of augmented Dickey-Fuller test after one level of difference

\begin{tabular}{|c|c|c|c|c|c|}
\hline \multirow{2}{*}{ State } & \multicolumn{3}{|c|}{ Critical Values } & \multirow{2}{*}{ Prob. } & \multirow{2}{*}{ t statistics } \\
\cline { 2 - 4 } & $1 \%$ & $5 \%$ & $10 \%$ & \\
\hline intercept and trend & -3.96 & -3.41 & -3.12 & 0 & -28.93 \\
\hline intercept & -3.43 & -2.86 & -2.56 & 0 & -28.94 \\
\hline Without intercept and trend & -2.56 & -1.94 & -1.61 & 0 & -28.88 \\
\hline
\end{tabular}

In last step, ARIMA $(1,0,0)$ used to forecast the exchange rate time series and the error of forecast is computed. The result can be seen in

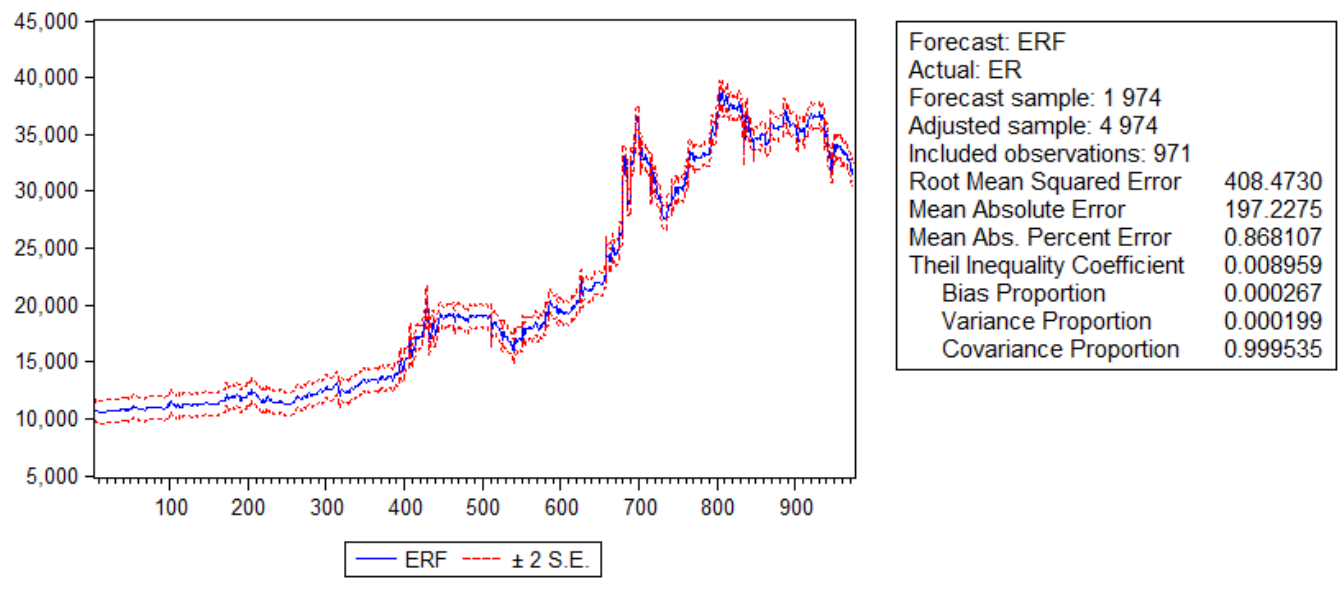

Figure 1.

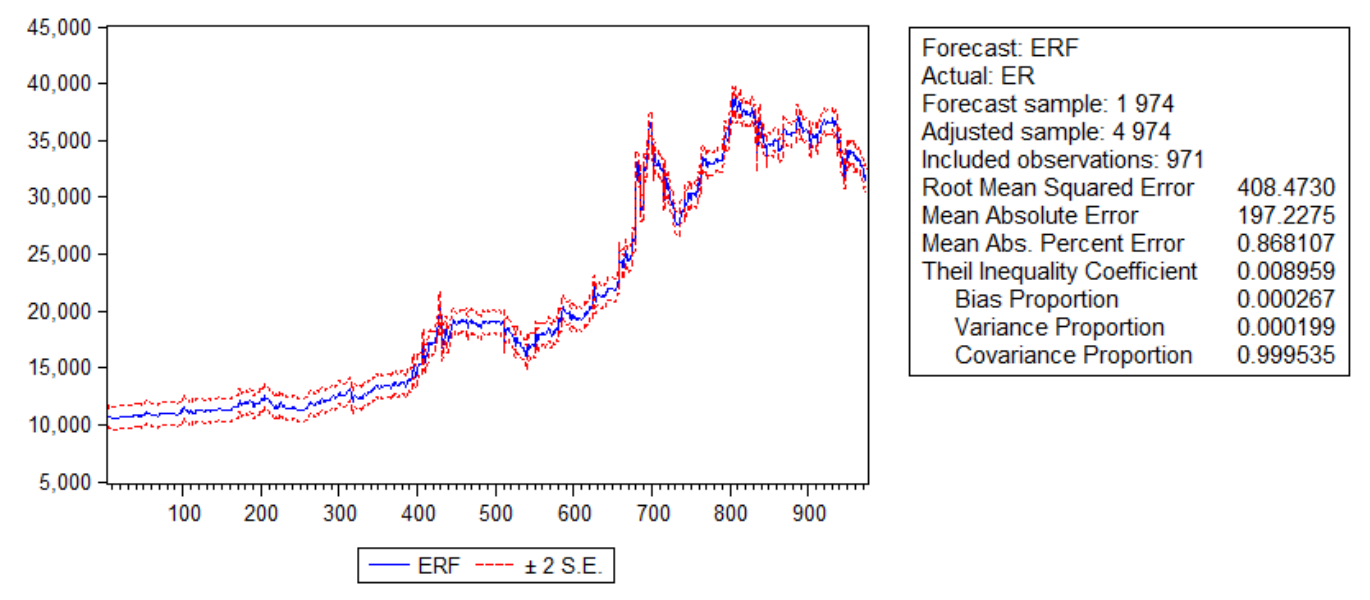

Figure 1. Forecast by ARIMA $(1,0,0)$ 


\section{A Brief Note on Artificial Neural Networks}

Basically artificial neural network is a tool for modeling unknown, complex or ambiguous process or systems. In such cases there is no full description of effective relationship between inputs and outputs or complexity of variables are so high that modeling is difficult via common approaches. Recently neural networks have been used for modeling non-linear economic relationship because of its ability to extract complex non-linear and interactive effects. Neural networks are a class of non-linear model that can approximate any non-linear function to an arbitrary degree of accuracy and have the potential to be used as forecasting tools in many different areas (Oyewale, 2013). The ability to process information and extract hidden knowledge from them and then generalize it to unsighted data, has turned the neural networks to powerful computational methods for modeling.

The inherently nonlinear structure of neural networks is useful for capturing the complexity underlying relationship in many real world problems. It is a more reliable methods for forecasting applications because not only they can find linear structure in a problem but they can also model linear processes. However, the weakness or limitations of neural networks in any study includes: Black-box problem which is a situation where all information are contained in weight matrices for their output and hidden layers, and these do not make apparent identities of salient predictor variables. This is in contrast with parametric variables which do not only identify influential variables but also provides the degree of their contributions. To overcome this problem, sensitivity analysis can be used to determine the effect and contribution of each input variable in output variable.

Usually there are some steps on designing a neural network which can briefly review as: neural network type selection, input variable selection, data collection, data processing, dividing data to training, testing and validation subsets, selection of the number of hidden layers, hidden neurons and output neurons, selection of transfer functions, choose of evaluation criteria and setting the of number of training iteration and choosing training algorithm.

Obviously there are a lot of different network parameters, and the configurations must be choose based on the application and conditions so determination of a good configuration for a neural network is a very complex and time consuming job and need some degree of experience and knowledge. It is important to note that after a proper network selection, it can be used in same problem but different data sets.

In the first and important step of every neural network, it should be trained. For this purpose usually the whole data set must be divided to training and testing subsets (or training, cross validation and testing subsets). Training subset is used for learning while test one is used for evaluation of network generalization ability in the end of training.

Cross validation test which usually used in sophisticated and complex training methods, is a measure of network training accuracy in each iteration while network is being trained. Although it may add some more computational cost, but it is able to find the correct direction of learning and lead the network training method to a better final point. Selection of good samples to add to cross validation and test subsets has a vital effect on learning accuracy of network. It's 


\section{Macrothink}

Business and Economic Research

ISSN 2162-4860

2014, Vol. 4, No. 2

a common mistake to use the last $\mathrm{x}$ percent of data as a test subset. This kind of mistake has a huge effect in long-term time series with high dynamic volatilities and the effect in short-term time series is not neglect able.

The testing subset's size is ranging from $10 \%$ to $30 \%$ of the data set. From empirical results it can be said that as a rule of thumb one hidden layer is enough to approximate any continuous function and using more than 2 hidden layers will not add any improvement to the network results.

In non-linear and complex situations, the problem of trapping in local minimums of error arises. In these cases by adding some more computational cost -usually a lot more-, it's possible to employ genetic search or optimization algorithms as a natural way of finding global minimum of error to reach the lowest possible amount of error.

Other parameters usually must be chosen via try and error or heuristically.

\section{Technical Approach}

A time delayed feed forward (TLFN) network which is a multilayer perceptron (MLP) with one hidden layer and memory elements used in this research. As the network must be worked on a time series, these memory elements let the network to learn the relationship between data over the time. The count of memory elements is relate to optimized time lag which represent the width of a window which cover all time related samples in time series. Training is unsupervised and the method of training is back-propagation of errors based on gradient decent algorithm. The time series is same as what is used in ARIMA model estimation in previous section.

\section{Performance Criteria}

In order to evaluate and compare accuracy of forecast results, the following performance measures are used and evaluated respectively: root mean square error (RMSE) and mean average error (MAE) functions. RMSE, and MAD are measures of the deviation between the actual and forecasted value. They can be used to evaluate forecasting error. The smaller the values of the criteria, the closer the forecasted value to the actual value.

$$
\begin{gathered}
\text { RMSE }=\sqrt{\frac{\sum\left(P_{t}-A_{t}\right)^{2}}{N}} \\
M A E=\frac{\sum\left|P_{\mathrm{t}}-A_{\mathrm{t}}\right|}{N}
\end{gathered}
$$

$p_{t=}$ The estimated amount of exchange rate.

$A_{t=}$ The real amount of exchange rate.

$\mathrm{N}=$ the number of estimated data. 


\section{Macrothink}

Business and Economic Research

ISSN 2162-4860

2014, Vol. 4, No. 2

The comparison results of these criteria has been summarized in the follow table:

Table 3. The result of two model accuracy

\begin{tabular}{|c|c|c|}
\hline & RSME & MAE \\
\hline Neural network & 214.7579 & 101.5478 \\
\hline Box-Jenkins & 408.4730 & 197.2275 \\
\hline
\end{tabular}

According to the table, accuracy of neural network is better than ARIMA model. The error is about the half in both measures. It means that artificial neural networks are better than common econometric models in modeling and forecasting exchange rate in this approach.

\section{Fundamental Approach}

In this section the neural network is used to estimate a model to forecast exchange rate variable based on other economic variables. It is obvious that this variable is influenced by many qualitative and quantitative economic variables.

Input variables are monthly data values of gold price, oil price, consumer price index in Iran and America over the period of Jan 2003 to June 2013, totally 128 data packs.

The trend of price index in Iran and America is shown. Of course the variables has been standardized to be in the same range.

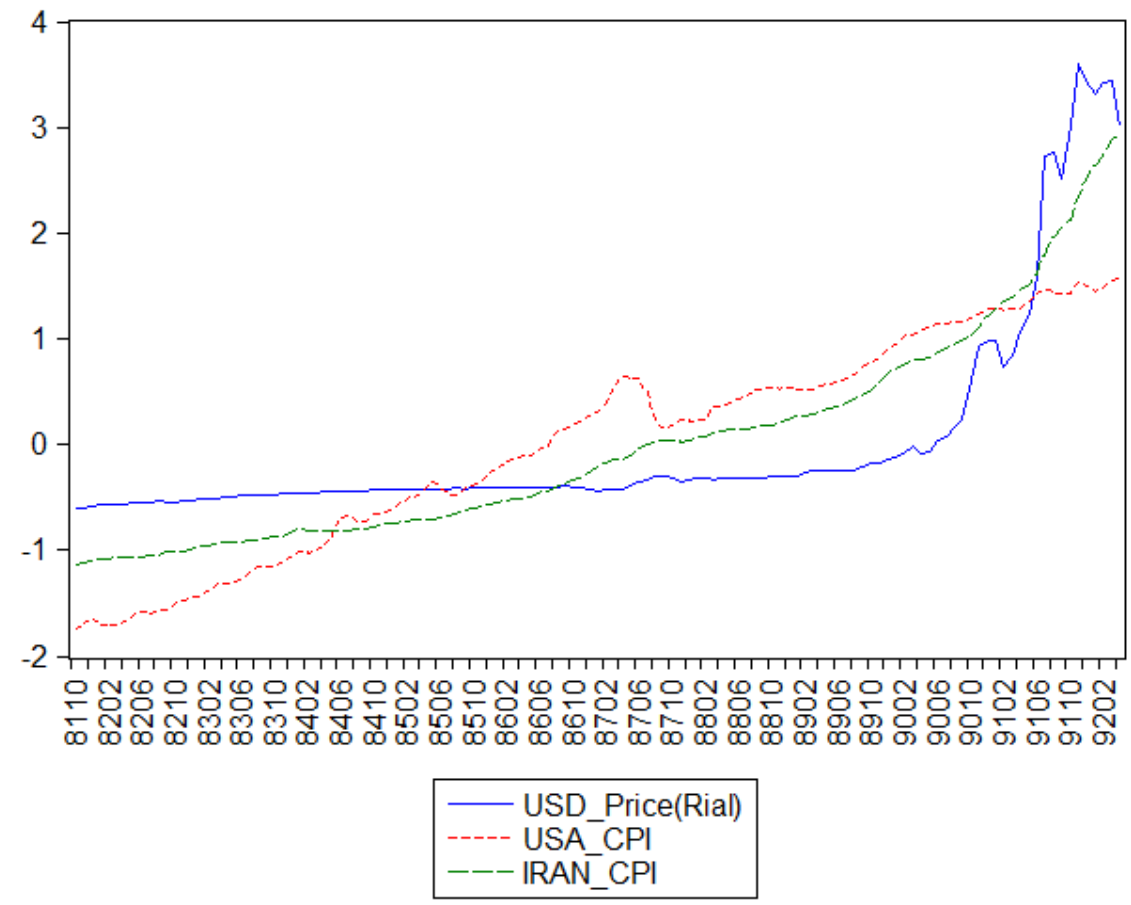

Figure 2. The trend of exchange rate, CPI in Iran \& America

By comparing the historical data of Iran and USA CPIs, the maximum of Iran CPI is 6.5 times of its base value while it is 1.3 for USA one. Hence if the network had been trained just based on price index of two countries, the exchange rate would had been very greater than 3 times in the end while the real factor is about 3 , so it means that other variables have been involved. 


\section{Al Macrothink}

Business and Economic Research

ISSN 2162-4860

2014, Vol. 4, No. 2

Hence the addition of some other variables to network inputs is logical. Here global gold price and Iran's crude oil price is chosen to add to network. Of course there are many other probably effective variables such as non-oil import and export, monthly GDP, foreign borrowing, future expectations, economic policies, balance of payments, economic crises and sanctions which are necessary to add, but because of inaccessibility to historical monthly data, they excluded from the network.

The following table show the gold and oil price trend to exchange rate in the period of time.

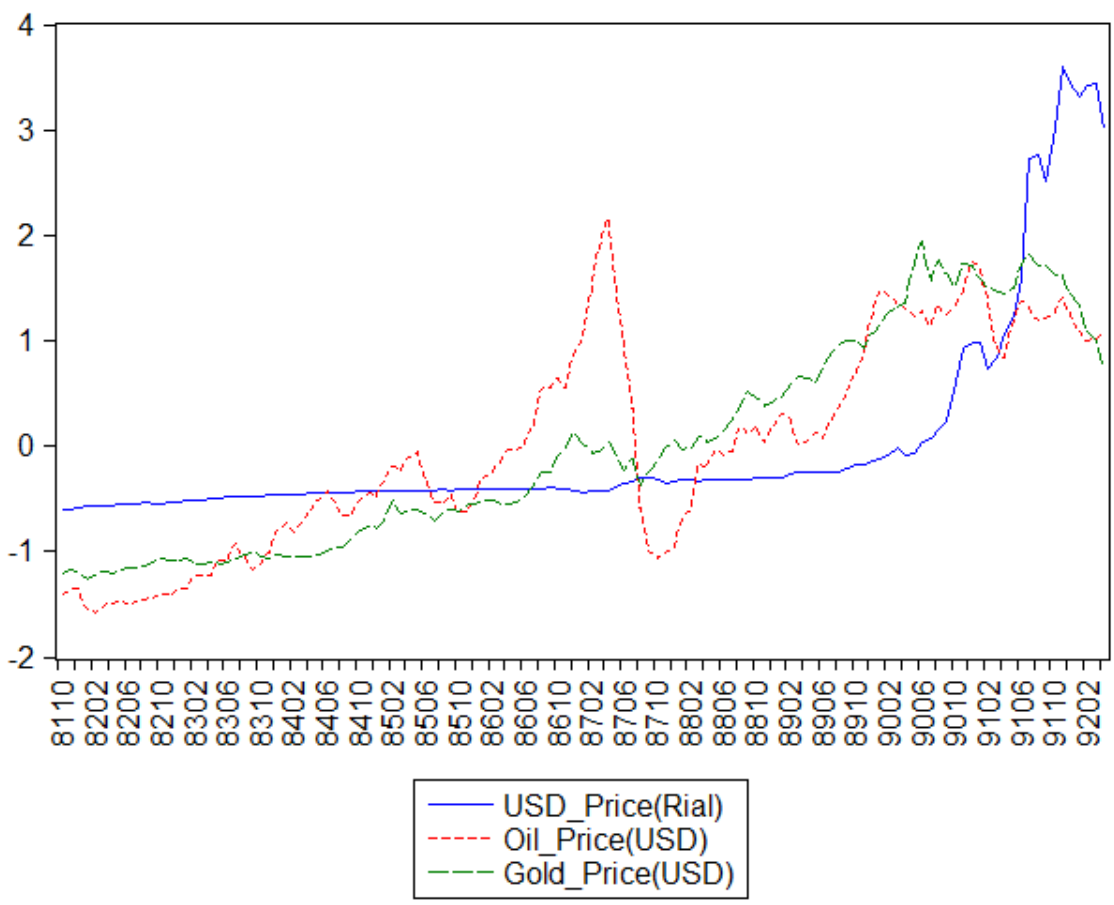

Figure 3. the trend of USD, oil and gold prices

The network is multilayer perceptron with one hidden layer again but without memory elements. Because in this approach the focus is on the relation between different variables, in order to simplify the situation and have a time independent series, arrangement of data is changed in a random basis. As the time based information is loosed, there is no need to have memory element here.

Training is unsupervised and the method of training is back-propagation of errors based on gradient decent algorithm again. Basically because of the nature of gradient descent method, the probability of being trapped in a local minimum is very high. While the purpose of training is to find the global minimum. In order to have a more optimized network, avoid of entrapment in local minimums and achieve the lowest error, a genetic search algorithm is used. Genetic algorithms act based on natural selection rule which means strong options will be transferred to next generation and the weak ones be eliminated. There are different genetics search methods in neural networks, here Generational G.A method is used. 


\section{MInstitute Macrothink $_{\text {Int }}$}

Business and Economic Research ISSN 2162-4860 2014, Vol. 4, No. 2

Genetic algorithms belong to the larger class of evolutionary algorithms (EA), which generate solutions to optimization problems using techniques inspired by natural evolution, such as inheritance, mutation, selection, and crossover.

In a genetic algorithm, a population of candidate solutions (called individuals, creatures, or phenotypes) to an optimization problem is evolved toward better solutions. Each candidate solution has a set of properties (its chromosomes or genotype) which can be mutated and altered; traditionally, solutions are represented in binary as strings of $0 \mathrm{~s}$ and $1 \mathrm{~s}$, but other encodings are also possible.

Here, the population parameter value is set to 25 and the number of generation's parameter is set to 100 . The results is shown in the following table.

Table 4. comparison the result of ANN to real values

\begin{tabular}{|c|c|c|c|c|c|c|}
\hline Time & \multicolumn{4}{|c|}{ Network input } & Real values & Network output \\
\hline Year/month & $\begin{array}{c}\text { Price index } \\
\text { in Iran }\end{array}$ & $\begin{array}{c}\text { Price } \\
\text { index in } \\
\text { USA }\end{array}$ & $\begin{array}{c}\text { Oil } \\
\text { price(\$) }\end{array}$ & $\begin{array}{c}\text { Gold } \\
\text { price(\$) }\end{array}$ & $\begin{array}{c}\text { Exchange } \\
\text { rate(Rial) }\end{array}$ & $\begin{array}{c}\text { Exchange } \\
\text { rate(Rial) }\end{array}$ \\
\hline 9012 & 390.11 & 125.38 & 122.52 & 1712.37 & 18926.55 & 20358.3552 \\
\hline 8405 & 137.81 & 107.39 & 56.02 & 435.41 & 8998.39 & 8936.148731 \\
\hline 9106 & 440.64 & 126.63 & 111.53 & 1712.84 & 23090.97 & 27617.01102 \\
\hline 8702 & 217.67 & 117.86 & 113.92 & 885.02 & 9178.06 & 9291.698272 \\
\hline 8902 & 273.85 & 118.96 & 78.77 & 1187.03 & 10206.65 & 10118.75427 \\
\hline 9105 & 428.98 & 125.97 & 106.24 & 1608.82 & 20603.23 & 23809.44097 \\
\hline 8806 & 258.66 & 118.23 & 68.84 & 984.64 & 9894.74 & 9948.356885 \\
\hline 8110 & 100.00 & 100.00 & 30.25 & 349.98 & 7980.93 & 7984.150095 \\
\hline 8809 & 263.60 & 119.04 & 74.29 & 1154.02 & 9948.50 & 9962.193171 \\
\hline 8603 & 175.97 & 113.49 & 66.35 & 658.41 & 9266.42 & 9334.149086 \\
\hline 8506 & 155.12 & 111.06 & 62.11 & 605.36 & 9190.42 & 9173.20965 \\
\hline 8710 & 244.52 & 116.07 & 39.73 & 845.13 & 9890.20 & 9827.765765 \\
\hline 9204 & 606.71 & 127.78 & 103.06 & 1263.21 & 33030.65 & 34035.02403 \\
\hline
\end{tabular}

According to above table, the amount of estimated exchange rate is very near to real ones, so the result of network has high accuracy. Although the difference in some cases is due to not access to all of effective variables. Nevertheless the results are satisfying and it is one of the neural networks advantages.

\section{Sensitivity Analysis in the Fundamental Network}

Sensitivity analysis is the study of how the uncertainty in the output of a mathematical model or system can be apportioned to different sources of uncertainty in its inputs (Saltelli et. al. 2008). Quite often, some or all of the model inputs are subject to sources of uncertainty, including errors of measurement, absence of information and poor or partial understanding of the driving forces and mechanisms. This uncertainty imposes a limit on our confidence in the response or output of the model. Further, models may have to cope with the natural intrinsic variability of the system, such as the occurrence of stochastic events (Der Kiureghian, et. al. (2009).

There are many different methods of sensitivity computations which usually be chosen by 
application, here a neural network adapted method is used.

Sensitivity of exchange rate to corresponding network inputs is computed. The results are given in the following table. It should be noted that the changes applied to standardized variables and the sensitivity values are in percent such that the sum of the values of each column is 100 .

Table 5. The result of exchange rate sensitivity to the input variables changes

\begin{tabular}{|l|c|c|c|c|}
\hline & 0.01 & 0.05 & 0.1 & 0.5 \\
\hline Iran's CPI & 26.99 & 24.87 & 22.81 & 12.18 \\
\hline USA's CPI & 47.40 & 49.59 & 51.48 & 56.88 \\
\hline Oil price & 8.67 & 8.09 & 7.66 & 6.63 \\
\hline Gold price & 16.92 & 17.43 & 18.03 & 24.30 \\
\hline
\end{tabular}

According to the high sensitivity of network to the USA price index, it sounds that it's a fake mathematic sensitivity because of small range of its changes. In other words according to 30 percent changes in whole data, the network hasn't trained by the out of this range data and so is very sensitive to its changes. In this regard other statistics seem illogical. To study more, USA's CPI variable has deleted, the network trained again and the sensitivity computed. The result is shown in the table 6.

Table 6. the result of exchange rate sensitivity to the input variables changes after deleting America CPI

\begin{tabular}{|l|c|c|c|c|}
\hline & 0.01 & 0.05 & 0.1 & 0.5 \\
\hline Iran's CPI & 51.58 & 52.83 & 54.20 & 56.23 \\
\hline Oil price & 20.55 & 20.94 & 21.96 & 24.91 \\
\hline Gold price & 27.86 & 26.22 & 23.82 & 18.85 \\
\hline
\end{tabular}

Now, after removing unnecessary variable, the weight of each input variable and the amount of its influence has determined. As reported by these table, Iran CPI is an effective factor in exchange rate. Also the model is sensitive to gold price more than oil price which is unimaginable in first sight. It may be due to the lack of their weights as input to the network. It seems if quantity of the monthly sells of oil and the import and export of gold historical data are accessible and apply to the network, more accurate and understandable results would be obtained.

In other hand, gold price may really has more contribution on exchange rate than crude oil. It may be due to some indirect effects. As a brief explanation, crude oil price may play a large part of its role via Iran's CPI indirectly. In addition, because Gold is possible to be trade as a commodity inside the country while crude oil is not, it induce both internal and external effects on exchange rate, while crude oil effect always must be pass through governmental filters to influence on exchange rate.

Thus via sensitivity analysis, the unnecessary input variable has deleted from the model and the real importance of each remained variable has investigated.

\section{Summary and Conclusions}

Modelling and forecasting exchange rate is usually carried out by the regression technique in 
economic researches. One short-come of this technique is data analyzed often exhibit some degrees of non-linearity that cannot be captured by a linear model. In this sense, Artificial Neural Network (ANN) which is highly flexible in estimation of non-linear models is a good candidate as an alternative for traditional methods.

Another issue is in the roadmap which is Box-Jenkins methodology. It's highly supervised and need an economy expert to be statistically trained to go through its steps to fit a good model to provided data. In this sense ANN is a good alternative again, with new effective training algorithms and optimized global minimum search methods and powerful computer hardwares available now, ANN modelling can be done completely unsupervised.

According to the results of first section of this research, while ARIMA estimated model is good in prediction, it cannot predict the value of exchange rate as accurate as possible. The comparison between ANN and ARIMA shows that the chosen ANN is far better, the ANN error is approximately the half of ARIMA in both measures.

Other advantages of ANN are short term and long term applicability of them, and the ability to be used in both technical and fundamental approaches. Generally these networks have facilitated forecasting and it's very suitable for end users.

In the second section, ANN is used in a long term approach to estimate a fundamental model for exchange rate. Four economic variables (price index in Iran and USA, Iran's crude oil price and global gold price) are chosen as inputs.

The results of network shows that while the overall error is very low and in most of the dates the network output is very close to real values but in some other ones it's obvious that the error quantity is high, which can be explained mostly by data limitation. However overall network performance indicate the ability of estimation of complex models even by access to a limited subset of data.

It should be noted that due to data limitation, reaching to a global minimum of error may be not simply while in this kind of situations genetic based search algorithms are very effective.

And the last point is the importance of sensitivity analysis of the trained network to the input variables. While it's obvious if the play range of an input variable is small, the sensitivity to that variable is so high, in this situation, sensitivity analysis can help to make a quantitative big number for that which will be a fake high mathematical sensitivity. In contrary, when there's a very low sensitivity to an input variable, which means the given variable has a low contribution to model, this analysis produce a very small number, and it can be interpreted as a noise or error in computation. Finally in a situation that a variable really has an important effect on model, this analysis will make a relatively acceptable number. Based on the resulted number any network designer is able to decide to include such variable to network or exclude it or to guess if he needs to add other missing variables to network.

So this approach can help to find and delete non-significant parameters from the model and purify it. While it is a useful tool for model designers, in the end, the weight and importance of each input variable is found. So policy makers are able to manage and control variables 
effective on exchange rate according to the contribution of them.

As a suggestion for next step in this research, it can be helpful to merge both methods and make a neural network which use past observations of exchange rate as an input to the network in addition to crude oil price and global gold price and Iran's CPI values. According to information theory, because the past values of exchange rate has information about all of related variables past values, it may fill the place of missed and inaccessible important economic variables in network.

Usually an important part of information is hidden in time relations in any time series. In second section of this research for simplicity and to focus on variable casual relations, those information is neglected and while the network results are satisfying, as a suggestion it's possible to enhance it by taking into account those information via adding memory elements to the network as it's done in first section of this research.

\section{Endnotes}

This article is based on a part of M.S. thesis of Mrs. Maryam Ebrahimi in Al-Zahra University of Tehran under supervision of Dr. Mehdi Pedram.

\section{References}

Akintola, K. G. Alese B. k., \& Thompson A. F. "Time series forecasting with neural networks: A case of stock prices of intercontinental blank Nigeria". IJRRAS, 9, 3.

AMM. Jamal. (2011). Modeling Exchange Rates with Neural Networks. Journal of Applied Business Research. 14, 1

C. A. Wan Khade, R. Mahalle, S., Gajbhiye, S., \& Bondade V. M. (2010). Use of the ARIMA Model for forecasting pigeon pea production in India. International Review of Business and Finance, 2, 97-102.

Chang, C. W., \& Foo, C. (2002). Forecasting the Volatility of Stock Indices: Using Neural Networks. Proceedings of Asia Pacific Economics and Business Conference, 2002(2), 919-928.

Der Kiureghian, A., Ditlevsen, O. (2009). Aleatory or epistemic? Does it matter? , Structural Safety, 31(2), 105-112. http://dx.doi.org/10.1016/j.strusafe.2008.06.020

Georgios Sermpinis (2012). Forecasting foreign exchange rates with adaptive neural networks using radial-basis functions and particle swarm optimization. Innovative Applications of O.R. 12.

Hu, Y. M., Zhang, G., Jiang, C. X., \& Patuwo, B. E. (1999). A Cross Validation Analysis of Neural Network Out-of-Sample Performance in Exchange Rate Forecasting. Decision Sciences, 30(1), 197-216. http://dx.doi.org/10.1111/j.1540-5915.1999.tb01606.x

Kamruzzaman, J, \& Sarker, R. (2003). Comparing ANN based on models with ARIMA for prediction of exchange rates. ASBOR Bulletin. 22, 2. 


\section{IIMacrothink}

Business and Economic Research ISSN 2162-4860 2014, Vol. 4, No. 2

Keck, A., A. Raubold, \& A. Truppia. (2009). Forecasting International Trade: A Time Series Approach, OECD Journal: Journal of Business Cycle Measurement and Analysis, 9(2009), 157-176.

P. G. Zhang. (2003). Time series forecasting using a hybrid ARIMA and neural network model. Neurocomputing. 50, 159-175. http://dx.doi.org/10.1016/S0925-2312(01)00702-0

Pradhan, R. (2010). Forecasting exchange rate in India: An application of artificial neural network model. Journal of Mathematics research, 2(4), 111-117. http://dx.doi.org/10.5539/jmr.v2n4p111

Saltelli, A., Ratto, M., Andres, T., Campolongo, F., Cariboni, J., Gatelli, D. Saisana, M., \& Tarantola, S., (2008). Global Sensitivity Analysis. The Primer, John Wiley \& Sons.

Shadboth, J., \& Taylor, J. (2002). Neural networks and the financial markets. Springer publishing company Limited.

Stock, J. H., \& M. W. Watson. (2003). Introduction to Econometrics. Addison Wesley.

Stokes, A. (2011). Forecasting exchange rates using neural networks: a traders approach. Student Theses \& Publication, 205.

Timmermann, A., \& Granger, C. (2004). Efficient Market Hypothesis and Forecasting. International Journal of Forecasting, 20(1), 15-27. http://dx.doi.org/10.1016/S0169-2070(03)00012-8

Vincenzo Pacelli. (2012). Forecasting Exchange Rates: A comparative analysis. International Journal of Business and social science. 3(10), 145-156.

\section{Copyright Disclaimer}

Copyright for this article is retained by the author(s), with first publication rights granted to the journal.

This is an open-access article distributed under the terms and conditions of the Creative Commons Attribution license (http://creativecommons.org/licenses/by/3.0/). 